\title{
APROXIMACIÓN DIAGNÓSTICA HISTOPATOLÓGICA A LA CARDIOMIOPATÍA CHAGÁSICA CRÓNICA EN Saguinus leucopus DENTRO DE PROGRAMAS DE REHABILITACIÓN Y REINTRODUCCIÓN
}

\author{
HISTOPATHOLOGICAL DIAGNOSTIC APPROACH \\ IN Saguinus leucopus WITH CRONIC CHAGASIC \\ CARDIOMYOPATHY IN REHABILITATION AND \\ RE-INTRODUCTION PROGRAMS
}

\author{
Edward Javier Acero-Mondragón¹, María Inés Maldonado-Arango², Nohora Mercedes Angulo-Calderón³
}

\begin{abstract}
1 Lic. Biología, Médico Veterinario, Esp. Laboratorio Clínico. M.Sc. (c) Morfología Humana. Profesor Facultad de Medicina Universidad Militar "Nueva Granada". Grupo Morfología Médica. Dirección: Transversal 5 No 99-00. Correspondencia: edward.acero@ unmilitar.edu.co ${ }^{2}$ Médico Cirujano, Esp. Educación Médica. M.Sc. (c) Morfología Humana. Profesora Asistente Facultad de Medicina Universidad de La Sabana. Grupo Proseim. Correspondencia: maria.maldonado@unisabana.edu.co 3 Médico Cirujano, Esp Anatomía Patológica. Profesora Asistente Facultad de Medicina Universidad de La Sabana. Correspondencia: nohora.angulo@ unisabana.edu.co
\end{abstract}

Rev. U.D.CA Act. \& Div. Cient. 15(1): 43 - 48, 2012

\section{RESUMEN}

Saguinus leucopus es un primate endémico de Colombia considerado vulnerable, además, con la distribución geográfica más restringida entre Saguinus sp. Así mismo, es reconocido como reservorio natural de Trypanosoma cruzi, por compartir la distribución geográfica con vectores, como Rhodnious prolixus y Triatoma dimidiata; sin embargo, el primate no presenta cardiomiopatía chagásica crónica de manera natural. Aproximaciones histopatológicas para diagnosticar cardiomiopatía chagásica crónica en $S$. leucopus, se deben establecer cuando en programas de rehabilitación y de reintroducción se hallen en los cuadros hemáticos Trypanosomas spp., paralelamente con decesos en las mismas colonias y que en las necropsias se demuestre cardiomegalias y esplenomegalias. Allí, la histopatología en corazónpuededemostrarmiocarditis, circunscritaalmiocardio con infiltrado linfomonocitario inmunifenotipificado CD 19 negativo, pero CD3 y CD68 positivo, hallazgos que, junto a los cardiomiocitos conteniendo formas bastoniformes de 2 micras de diámetro, encerradas en una "vaina" eosinófila de grosor homogéneo, son característicos de "pseudoquistes" amastigóticos miocárdicos de cardiomiopatia chagásica, propios de los causados por la especie $T$. cruzi.

Palabras clave: S. leucopus, T. cruzi, cardiomiopatia chagásica.

\section{SUMMARY}

Saguinus leucopus is an endemic primate in Colombia considered vulnerable, furthermore with the most restricted geographical distribution between Saguinus spp.. It is known as a natural reservoir of Trypanosoma cruzi since it shares geographic distribution with the vectors Rhodnious prolixus and Triatoma dimidiate. However, this primate does not develop chronic chagasic cardiomyopathy in a natural way. Nevertheless, a histopathological chronic chagasic cardiomyopathy approach diagnosis should be established in S. leucopus in rehabilitation centers and for reintroduction programs, when they have been diagnosed to be infected by Trypanosoma spp. with haemograms. Simultaneously, when death occurs in the same colony and necropsy shows cardiomegaly and splenomegaly. In these cases, heart histopathology could display myocarditis with lymphomonocitary infiltration, limited to myocardium, with negative CD 19 but positive CD3 and CD68. These findings, together with the observation of cardiomyocytes containing rod-forms of 2 microns in diameter, enclosed into a "pod" with homogeneous eosinophilic thickness are characteristic of "pseudocysts", the typical amastigotes in "Chagasic cardiomyopathy" caused by $T$. cruzi.

Key words: S. leucopus, T. cruzi, chagasic cardiomyopathy. 


\section{INTRODUCCIÓN}

Saguinus leucopus (Gunter, 1877) (Primates, Cebidae) es una especie de primate endémico de Colombia (HernándezCamacho \& Cooper et al. 1976; Defler, 2003). Su distribución geográfica es la más restringida entre los Saguinus spp. (Hernández-Camacho \& Cooper, 1976; Emmons, 1997), hallándose en el norte de Colombia, entre los ríos Magdalena y Cauca (Defler, 2003). Por disminución dramática de su hábitat asociado a deforestación (Poveda \& SánchezPalomino, 2004) es considerado como vulnerable, por la Unión Internacional para la Conservación IUCN y el apéndice I del Convention International Trade in Endangered Species of Wild Fauna and Flora CITES (CITES, 2001). S. leucopus es un primate reservorio natural Trypanosoma cruzi (Chagas, 1909) (Kinetoplastida. Trypanosomatidae) al compartir distribución geográfica con los vectores Rhodnious prolixus (Sthal, 1852) (Hemiptera, Reduviidae) y Triatoma dimidiata (Latreille, 1811) (Hemiptera, Reduviidae) (WHO 2002). Saguinus spp. como otros primates pequeños del nuevo mundo siendo reservorios, no presentan cardiomiopatía chagásica de manera natural (Teixeira et al. 2006), aunque sí han sido logradas en infecciones experimentalmente, en fase aguda, para otros primates neotropicales, como Cebus apella (Linnaeus, 1758) (Primates, Cebidae) (Teixeira et al. 2006; Almeida et al. 1992; Rosner et al. 1988) y, en fase crónica, después de un año de la infección en $C$. apella, Callitrix penicilata (É. Geoffrey, 1812) (Primates, Callitrichidae) y Saimiri sciureus (Linneo 1758) (Primates, Cebidae) (Teixeira et al. 2006). Sobre Saguinus spp. en cautividad, la literatura reporta aproximaciones diagnósticas a las cardiomegalias por cardiomiopatía dilatada que llevan a falla cardiaca de forma espontánea, describiéndose histopatológicamente fibrosis cardiacas mínimas (David et al. 2009), similar a las logradas experimentalmente en colonias cautivas de Saguinus spp., infectadas con virus de la Hepatitis GB (Chamanza et al.2006); sin embargo, las caracterizaciones anatomopatológicas de cardiomiopatía con cardiomegalia asociada a enfermedad de Chagas, con borramiento del ápice del ventrículo izquierdo, típico del hallazgo anatomopatológico de la cardiomiopatía chagásica crónica en humanos, solo se ha descrito en un tercio de los primates del nuevo mundo, cuando son infectados experimentalmente (Teixeira et al. 2006); en S. leucopus cautivos no hay antecedentes histopatológicos dirigidos a la búsqueda de cardiomiopatía chagásica crónica, cuando se encuentran cardiomegalias en las necropsias; por consideraciones epidemiológicas de la enfermedad de Chagas, el objetivo de este articulo es plantear una aproximación histopatológica al diagnóstico de cardiomiopatías chagásicas crónicas en las cardiomegalias halladas en las necropsias de las especies de S. leucopus, en programas de rehabilitación y de reintroducción

\section{MATERIALES Y MÉTODOS}

En los programas de rehabilitación y de reintroducción de S. leucopus, los animales fallecidos en colonias de esta especie, cuando se les practique necropsia y en ella se encuentre organomegalia cardiaca y esplénica, se les deben extraer estos órganos, fijándolos con formol al $10 \%$. En un laboratorio de Patología, se deben hacer cortes transversales del ápice cardiaco y del diámetro esplénico mayor, submuestreando segmentos de corazón y del bazo, con tamaños de $0,5 \mathrm{~cm}^{3}$, para procesamiento histotécnico y cortes en parafina a 3 micras; los cortes obtenidos, se deben colorear con histoquímica de Hematoxilina y de Eosina; las láminas histológicas obtenidas, se deben observar con microscopio óptico de luz en objetivo de $5 \mathrm{X}$ y 10X, buscando en corazón, pérdida de la arquitectura de las fibras miocárdicas con fibrosis y en endomisio infiltrados linfomonocitarios circunscritos (Arboleda \& Quintana, 2007; Barrera et al. 2008); paralelamente, se debe buscar cardiomiocitos conteniendo múltiples formas bastoniformes de color basófilo, las cuales, a objetivo de 40X y 100X con micrómetro, se deben apreciar con diámetro aproximado de 2 micras (Teixeira et al. 2006; Almeida et al. 1992; Rosner et al. 1988). Por técnicas inmunohistoquímicas, el infiltrado linfomonocitario miocárdico, se debe inmunofenotipificar buscando clusters de diferenciación CD3, CD19 y CD68 (Barrera et al. 2008; Arboleda \& Quintana, 2007; Camargos et al. 2000). En los cortes de bazo coloreados con Hematoxilina y con Eosina y observados con objetivos de 5X y 10X, entre tanto, se buscan folículos linfoides y vainas linfáticas periarteriales con focos reactivos, en zona marginal e infiltrada, principalmente, por macrófagos (O’Daly et al. 1984).

\section{RESULTADOS Y DISCUSIÓN}

En Colombia, los programas de rehabilitación y de reintroducción practican cuadros hemáticos en S. leucopus cautivos y se han hallado hemoparasitosis por Microfilaria spp. (Ladino \& Moreno, 2007; Fox et al. 2008); así mismo, en Saguinus spp. silvestres, se ha demostrado Trypanosoma spp. (Hershkovitz, 1977). Particularmente en las hemoparasitosis por Trypanosomas spp. es difícil reconocer la especie $T$. cruzi, porque si bien los trypomastigotes sanguíneos se pueden distinguir morfométricamente de la especie $T$. minasense (Ziccardi \& Olivera, 1999) son mas difíciles de diferenciarse entre $T$. rangeli y $T$. cruzi (De Sousa, 1999) y solo $T$ cruzi causa miocardiopatia chasgásica (Maudlin et al. 2004), por tanto, el hallazgo de estos hemoparásitos por cuadro hemático en los procesos de rehabilitación de S. leucopus, no indican iniciar conductas terapéuticas para eliminarlos (De Sousa, 1999; Maudlin et al. 2004); mas aún, si desde el punto de vista epidemiológico en Colombia se 
hiciere por hemograma un diagnóstico Trypanosoma spp. en S. leucopus de centros de rehabilitación con altitudes como 2600 msnm (como en Bogotá-Colombia), también se hace inoficioso iniciar conducta terapéutica con fines de evitar iniciación de ciclos epidemiológicos, porque no existe en estas latitudes descritos vectores Rhodnious sp. y Triatoma sp., para Trypanosoma spp. (WHO, 2002).

Ahora, si en Colombia centros de rehabilitación con programas de reintroducción para $S$. leucopus se ubican por debajo de 1800 msnm y se hallan Trypanosomas spp. en cuadros hemáticos y es acompañado este reporte con muertes de colonias de estos primates, se deben practicar necropsias en busca de organomegalias en corazón y en bazo, iniciando, con estos hallazgos, el abordaje diagnóstico de cardiomiopatía chagásica crónca, la cual, está reportada de forma natural en mamíferos del hemisferio occidental, asociadas únicamente a la especie de $T$. cruzi (Teixeira et al. 2006; WHO 2002) y que por su habilidad de hacer fases amastigotas intracelulares en miocardio causando miocarditis chagásica, lo diferencia de T. rangeli (D’alessandro, 1974) o T. minasense (Ruivo et al. 2005).

La aproximación diagnóstica histopatológica a la cardiomiopatía chagásica crónica en S. leucopus dentro de programas de rehabilitación y de reintroducción, inicia con la demostración histopatológica en miocardio de focos circunscritos de infiltrado linfomonocitario compatibles con miocarditis (Foto 1a), alternándose con cardiomiocitos entre hipertróficos y normotróficos (Rosner et al. 1988; Camargos et al. 2000); en algunos cortes histológicos, se puede alternativamente observar múltiples formas bastoniformes de 2 micras de tamaño, aproximadamente, en cuyo interior sobresale una estructura basófila redondeada $\mathrm{o}$ puntiforme; todas las formas bastoniformes descritas, a su vez encerradas en una "vaina" eosinófila de grosor homogéneo, son compatibles con lo que se conoce como "pseudoquistes" amastigóticos de T. cruzi (Foto 1b) (Teixeira et al. 2006; Almeida et al. 1992; Rosner et al. 1988) y han sido observados y descritos por infección experimental de $T$. cruzi en C. apella (Teixeira et al. 2006; Almeida et al. 1992; Rosner et al. 1988), roedores (Camargos et al. 2000; Barrera et al. 2008) y en infecciones naturales crónicas en humanos (Arboleda \& Quintana, 2007). Todos estos hallazgos en miocardio, se deben acompañar histopatológicamente en el bazo esplenomegálico con folículos linfoides y vainas linfáticas periarteriales reactivas con focos en zona marginal nodular linfóidea, infiltrada, principalmente, por macrófagos (O’Daly et al. 1984) (Foto 1c).

Con respecto a la inmunohistoquímica que fenotipifíca el infiltrado linfomonocitario de la miocarditis es asociada a enfermedad chagásica crónica, cuando marca positivo CD3, para Linfocitos T (Parodi et al. 2009) (Foto 2a), negativa para Linfocitos B CD19 (O'Daly et al. 1984) (Foto 2b) y positiva para Macrófagos CD68 (Santander et al. 2007) (Foto 2c); combinación inmunohistoquímica demostrada en infecciones crónicas experimentales de $T$ cruzi en ratas (Camargos et al. 2000), en ratones (Barrera et al. 2008) y en aves (Teixeira et al. 2011) e infecciones crónicas naturales en humanos (Arboleda \& Quintana, 2007).

Dado que $T$. cruzi, $T$. rangeli y $T$ minasense están descritos en Saguinus spp. colombianos (Hershkovitz, 1977), los procesos de rehabilitación deben involucrar esfuerzos para confirmarlos taxonómicamente más allá del Género, ya que en Colombia no hay datos epidemiológicos de prevalencia de estos hemoparásitos, en Saguinus spp.

Un estudio panameño reporta para S. geoffroyi prevalencias de $1,3 \%$ para $T$. cruzi; $52,7 \%$, para $T$. minasense y $25 \%$ para T. rangeli (Sousa et al. 1974; Sousa \& Dawson, 1976); estos datos, se podrían compartir en Colombia, porque las áreas de distribución geográfica de S. geoffry (Emmons,



a.

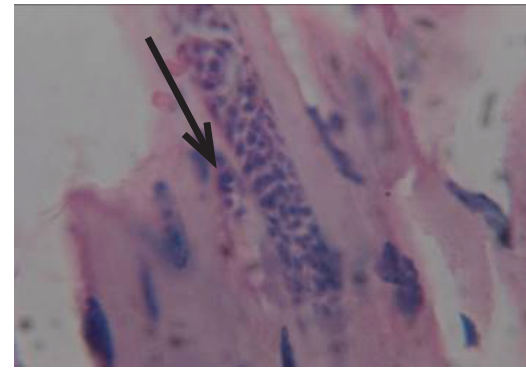

b.

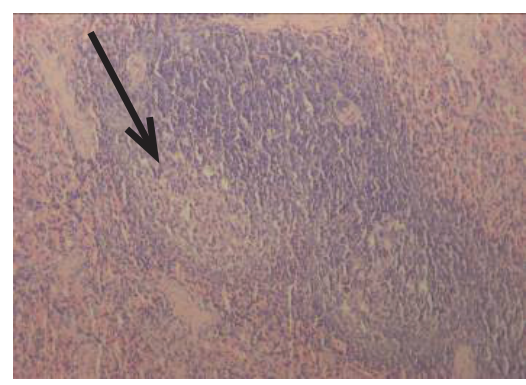

c.

Foto 1. a) Microfoco miocardico con infiltrado linfomonocitario. HEE 10 X; b) Cardiomiocito de trabajo con "Pseudoquiste" conteniendo formas amastigotas (señalados con la flecha) de Trypanozoma cruzi. HEE 100 X; c) Vaina linfática periarteriolar arriba con zona marginal (señalado con la flecha), infiltrada por macrófagos. HEE 10 X. 


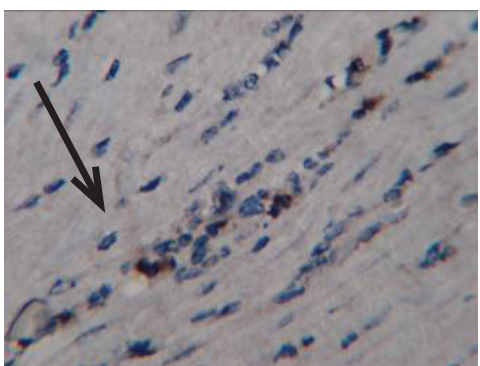

a.

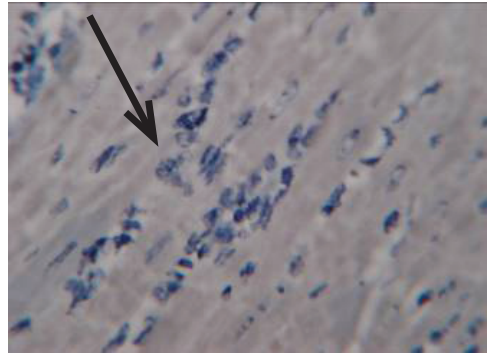

b.

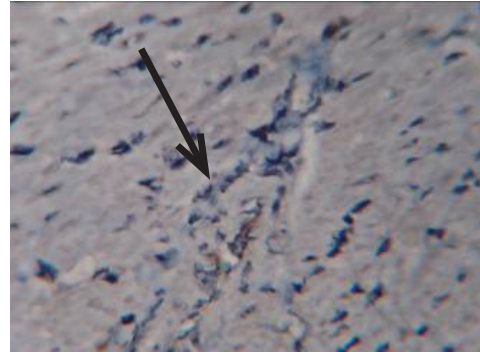

C.

Foto 2. Fenotipificación del infiltrado linfomonocitario de la miocarditis. a) Marcaje positivo para CD 3. Hematoxilina 10 X; b) Marcaje negativo para CD 19. Hematoxilina 10 X; c) Marcaje Positivo para CD68. Hematoxilina 10 X.

1997) están dentro de la misma área de distribución de los vectores de triatomidos $R$. prolixus y $T$. dimidiata, en el norte de Suramérica (WHO, 2002). Mas aun, si se hallan cardiomagalias asociadas a cardiomiopatía chagásica en $S$. leucopus, se pueden relacionar en nuestro medio el tropismo asociado a clones de T. cruzi tipo I o de ciclo selvático (Botero et al. 2007), que está demostrado, incluso, puede iniciar brotes chagásicos por vía de transmisión oral (Benchimol, 2006; Coura, 2006). Estudios epidemiológicos demuestran que $T$. cruzi tipo I o de ciclo selvático tienen distribución geográfica al norte de Suramérica, por arriba de la cuenca del amazonas (Miles et al. 2003) y que predominan en Colombia (Duque et al. 2011), coincidiendo en este territorio con las áreas distribución de S. leucopus (Defler, 2003; Emmons, 1997). En el contexto de salud pública, estudios de relación entre cardiomiopatía chagásica crónica en S. leucopus dentro de programas de rehabilitación y de reintroducción y la prevalencia de $T$. cruzi tipo I en la zona de distribución de $S$. leucopus, no han sido establecidos, considerando la dramática disminución de su hábitat (Poveda \& SánchezPalomino, 2004), con programas de reintroducción para esta especie de primate (Leal et al. 2010; Ruivo et al. 2005) presionados por ser el Cébido de más alto tráfico en la ciudad de Bogotá, Colombia (Ruiz-García et al. 2010); preguntas como cंcuáles son los riesgos de introducir $S$. leucopus sin diagnóstico de tripanosomiasis en poblaciones silvestres de $S$. leucopus y qué riesgos tiene la población humana en las zonas de reintroducción dentro de la dinámica reservorio vulnerable-vector? Se deben empezar a resolver.

Conflicto de intereses: Declaramos que el manuscrito fue preparado y revisado por los autores y no tienen ningún conflicto de intereses que ponga en riesgo la validez de los resultados presentados. Financiación: El estudio histopatológico e inmunohistoquímico de este trabajo fue financiado con recursos propios de los autores

\section{BIBLIOGRAFÍA}

1. ALMEIDA, E.A.; NAVARRO, M.R.; GUARIENTO, M.E.; CARVALHAL, S.S. 1992. Infecção experimental de macacos Cebus apella. pelo Trypanosoma cruzi. Avaliação clínica, eletrocardiográfica. Rev. Soc. Bras. Med. Trop. 25:7-12.

2. ARBOLEDA, F.; QUINTANA, H. 2007. Histopatología de la enfermedad de Chagas. En: Rosas, F.; Vanegas, D.; Cabrales, M. eds. Enfermedad de Chagas. Bogotá: Sociedad Colombiana de Cardiología y Cirugía Cardiovascular. 21p.

3. BARRERA, Y.K.; GUEVARA, J.M.; PAVÍA, P.X.; MONTILLA, M.; NICHOLLS, R.S.; PARRA, E.; PUERTA, C.J. 2008. Evaluación de las pruebas de PCR TcH2AF-R y S35-S36 para la detección de Trypanosoma cruzi en tejido cardiaco de ratón. Biomédica. Colombia. 28:616-626.

4. BENCHIMOL, P.R. 2006. The oral transmission of Chagas' disease: An acute form of infection responsible for regional outbreaks. Int. J. Cardiology. Holanda. 112:132-133.

5. BOTERO, L.A.; MEJÍA, A.M.; TRIANA, O. 2007. Caracterización biológica y genética de dos clones pertenecientes a los grupos I y II de Trypanosoma cruzi de Colombia. Biomédica. 27(1):64-74.

6. CAMARGOS, E.R.S.; FRANCO, D.J.; GARCIA, C.M.M.G.; DUTRA, A.P.; TEIXEIRA, A.L. Jr.; CHIARI, E. 2000. Infection with different Trypanosoma cruzi populations in rats: myocarditis, cardiac sympathetic 
denervation, and involvement of digestive organs. Am. J. Trop. Med. Reino Unido. 62(5):604-612.

7. CHAMANZA, R.; PARRY, N.M.A.; ROGERSON, P.; NICOL, J.R.; BRADLEY, A.E. 2006. Spontaneous lesions of the cardiovascular system in purpose-breed laboratory nonhuman primates. Toxicol Pathol. USA. 34:357-363.

8. CITES. 2001. Lista Cites. Una referencia a los Apéndices de la Convención sobre el Comercio Internacional de Especies Amenazadas de Fauna y Flora Silvestres. Compilada por el PNUMA Centro de Monitoreo de la Conservación Mundial. Con el concurso financiero de la Secretaría de la Convención sobre el Comercio Internacional de Especies Amenazadas de Fauna y Flora Silvestres, la Comisión Europea y el Joint Nature Conservation Committee. Printing Group Unwin Brothers, Martins, Old Woking, Surrey. SUIZA. 235-265. Disponible en: http://www.mzp.cz/www/ laws/cites2.nsf/ea65e91b58b6632cc12564df002c 44d8/0a2d18d902797bc1c125656e0050b9ea/\$FI LE/Checklists\%20on\%20CD-ROM.pdf (con acceso 16/06/2011).

9. COURA, J.R. 2006. Transmission of chagasic infection by oral route in the natural history of Chagas disease. Rev Soc Bras Med Trop. 39(Suppl 3):113-117.

10. D'ALESSANDRO, A. 1974. The problem of Chagas' disease in the Western hemisphere. Bol. Oficina Sanitaria Panamericana. Pan Am. San. Bureau. USA. 763:242- 247.

11. DAVID, J.M.; DICK. E.J. Jr.; HUBBARD, G.B. 2009. Spontaneous pathology of the common marmoset Callithrix jacchus and tamarins Saguinus oedipus, Saguinus mystax. J. Med. Primatol. USA. 38(5):347359.

12. DEFLER, T. 2003. Primates of Colombia. Conservación Internacional., Washington D. C. USA. 543p.

13. DESOUSA, M.A. 1999. Morphobiological characterization of Trypanosoma cruzi Chagas, 1909 and its distinction from other Trypanosomes. Mem. Inst. Oswaldo Cruz. Brasil. 94(1):205-210.

14. DUQUE, M.C; RAMÍREZ, J.D.; RENDÓN, L.M.; GUHL. F. 2011. Evaluación de la variabilidad genética de aislamientos colombianos de Trypanosoma cruzi mediante marcadores microsatélites. Infectio. Colombia. 15(4):227-234.
15. EMMONS, L. 1997. Neotropical Rainforest Mammals A Field Guide(2nd ed) Chicago, Ill ;London: Univ. of Chicago Press. Reino Unido. 110p.

16. FOX, M.; BRIEVA, C.; MORENO, C.; MACWILLIAMS, P.; THOMAS, C. 2008. Hematologic and serum biochemistry reference values in wildcaught whitefooted tamarins (Saguinus leucopus) housed in captivity. J. Zoo. Wildl. Med. USA. 39:548-557.

17. HERNÁNDEZ-CAMACHO, J.; COOPER, R.W. 1976. The nonhuman primates of Colombia. In: Thorington, R.W. Jr.; Heltne, P.G. (eds). Neotropical Primates: Field Studies and Conservation, National Academy of Sciences, Washington, D.C., USA. 35p.

18. HERSHKOVITZ, P. 1977. Living New World monkeys (Platyrrhini), with an introduction to Primates. University of Chicago Press, Chicago, USA. 751p.

19. LADINO DE LA H, R.; MORENO, O.M.I. 2007. Prevalencia de Microfilaria spp. en primates de zoológicos colombianos. Rev. Med. Vet. Colombia. 13:83-94.

20. LEAL, A.; GRANADOS, J.L.; ZERDA, E.; BRIEVA. C. 2010. Liberación y seguimiento de dos grupos de tití gris (Saguinus leucopus) en el departamento de Tolima. Rev. Med. Vet. Zoot. Colombia. 57:132-143.

21. MAUDLIN, I.; HOLMES, P. H.; MILES, M.A. 2004. The trypanosomiases. CABI Publishing. Reino Unido. 614p.

22. MILES, M.A.; FELICIANGELI, M.D.; DE ARIAS, A.R. 2003. American trypanosomiasis (Chagas disease) and the role of molecular epidemiology in guiding control strategies. BMJ. Reino Unido. 326:14441448.

23. O'DALY, J.A.; SIMONIS, S.; DE ROLO, N.; CABALLERO, H. 1984. Suppression of humoral immunity and lymphocyte responsiveness during experimental Trypanosoma cruzi infections..Rev. Inst. Med. Trop. Sao Paulo. Brasil. 26(2):67-77.

24. PARODI, C.; PADILLA, A.M.; BASOMBRIO, M.A. 2009. Protective immunity against Trypanosoma cruzi. Mem. Inst. Oswaldo Cruz. Brasil. 104(1):288-294.

25. POVEDA, K.; SÁNCHEZ-PALOMINO, P. 2004. Habitat use by the white-footed Tamarin, Saguinus leucopus: a comparison between a forest-dwelling group and an urban group in Mariquita, Colombia. Neotropical Primates. USA. 12(1):6-7. 
26. ROSNER, J.M.; SCHININI, A.; ROVIRA, T.; ARIAS, A.; VELÁSQUEZ, G.; IDALLA-MONZÓN, M. 1988. Acute Chagas' disease in non-human primates. Chronology of clinical events, clinical chemistry, ECG, radiology, parasitemia and immunological parameters in the Cebus apella monkey. Trop Med Parasitol. Alemania. 39:51-55.

27. RUIVO, E.B.; CARROLL, J.B.; MORALES-JIMÉNEZ, A.L. 2005. The silvery-brown Tamarin (Saguinus leucopus) conservation project. Neotropical Primates. USA. 13(3):36-39.

28. RUIZ-GARCÍA, M.; LEGUIZAMÓN, N.; VÁSQUEZ, C.; RODRÍGUEZ, K.; CASTILLO, M.I. 2010. Métodos genéticos para la reintroducción de monos de los géneros Saguinus, Aotus y Cebus (Primates: Cebidae) decomisados en Bogotá, Colombia. Rev. Biol. Trop. (Int. J. Trop. Biol.) Reino Unido. 58(3):1049-1067.

29. SANTANDER, S.P.; CUÉLLAR, A.A.; THOMAS, M.C.; GUZMÁN, F.; GÓMEZ, G.A.; LÓPEZ, M.C.; PUERTA, B.C. 2007. Expresión de marcadores en células dendríticas de pacientes chagásicos crónicos estimuladas con la proteína KMP-11 y el péptido K1 de Trypanosoma cruzi. Biomédica. Colombia. 27(1):18-27.

30. SOUSA, O.E.; ROSSAN, R.N.; BAERG, D.C. 1974. The preval trypanosomes and microfilariae in Panamanian monkeys. Am. J. Trop. Med. Hyg. USA. 23:862-868.
31. SOUSA, O.E.; DAWSON, G.A. 1976. Trypanosome infections in the marmoset Saguinus geoffroyi from the Panama canal zone. Am. J. Trop. Med. Hyg. USA. 25:407-409.

32. TEIXEIRA, A.R.L.; NASCIMENTO, R.J.; STURM, N.R. 2006. Evolution and pathology in Chagas disease: a review. Mem. Inst. Oswaldo Cruz. Brasil. 101(5):463491.

33. TEIXEIRA, A.R.L.; HECHT, M.M.; GUIMARO, M.C.; SOUSA, A.O.; NITZ, N. 2011. Pathogenesis of Chagas' Disease: Parasite Persistence and Autoimmunity. Clin. Microbiol. Reviews.24(3):592-630,

34. World Health Organization WHO. 2002. Control of Chagas disease. Second Report of the WHO Expert Committee. WHO Technical Report Series. USA. 905:1-109.

35. ZICCARDI, M.; OLIVEIRA, R.L. 1999. Polymorphism in trypomastigotes of Trypanosoma (Megatrypanum) minasense in the blood of experimentally infected squirrel monkey and marmosets. Mem. Inst. Oswaldo Cruz. 94(5):649-653.

Recibido: Julio 25 de 2011

Aceptado: Marzo 25 de 2012 